\title{
Evento extremo de chuva no Rio de Janeiro: análise sinótica, previsão numérica e comparação com eventos anteriores
}

\author{
Extreme precipitation episode in Rio de Janeiro: synoptic analysis, numerical \\ simulation and comparison among previous events \\ Kelen Martins Andrade ${ }^{1}$, Henri Rossi Pinheiro ${ }^{1}$ e Giovanni Dolif Neto ${ }^{1}$ \\ ${ }^{1}$ Centro de Previsão de Tempo e Estudos Climáticos/Instituto Nacional de Pesquisas \\ Espaciais (CPTEC-INPE, Cachoeira Paulista, Brasil
}

\begin{abstract}
Resumo
O objetivo deste trabalho é fazer uma análise sinótica e verificar a destreza de modelos numéricos operacionais (ETA15km, BRAMS20km e GFS) para um caso de chuva intensa em parte do litoral de São Paulo e do Rio de Janeiro, Baixada Fluminense e Região Serrana do Rio de Janeiro entre os dias 17 e 18/03/2013. Em algumas localidades a precipitação acumulada em 24 horas excedeu 100 mm, com máximo de 424,5 na cidade de Petrópolis. Foi usada uma ferramenta para a obtenção de padrões sinóticos ocorridos no passado semelhante ao evento extremo analisado. As fortes chuvas foram ocasionadas pela presença de uma frente fria associada aos ventos mais intensos em baixos níveis, favorecendo a advecção de umidade do oceano para o continente. Os modelos numéricos regionais representaram de forma qualitativamente adequada a chuva intensa na região atingida. Porém, a intensidade e a localização das chuvas intensas ainda é um desafio para a modelagem numérica.
\end{abstract}

Palavras-chave: Evento extremo. Modelo numérico. Previsibilidade.

\begin{abstract}
The purpose of this study is to analyze the synoptic pattern and to verify the skill of numerical models (ETA15km, BRAMS20km and GFS) for intense rainfall event in the coastal of São Paulo and Rio de Janeiro states, Baixada Fluminense and the Mountain Region of Rio de Janeiro in march 17-18, 2013. In some places the rainfall in 24 hours exceeded $100 \mathrm{~mm}$, reaching maximum of $424.5 \mathrm{~mm}$ in Petropolis. An objective tool was used for obtaining synoptic patterns occurred in the past similar to the extreme event studied. Heavy precipitation was caused by the presence of a cold front associated with intense low levels circulation, contributing for moisture advection from Atlantic Ocean toward the continent. The regional numerical models satisfactorily predicted the heavy rainfall episode. However, the intensity and location of heavy rainfall is still a challenge for numerical modeling.
\end{abstract}

Keywords: Extreme event. Numerical models. Predictability. 


\section{Introdução}

Eventos de chuva intensa, responsável por enchentes e deslizamentos de terra, normalmente causam diversos transtornos à sociedade e acarretam prejuízos sócio-econômicos. Tais eventos são comuns sobre o Sudeste do Brasil, especialmente na estação chuvosa. Porém, a particularidade de alguns eventos está no volume acumulado de chuva, que algumas vezes é considerado extremo. No decorrer do dia 17/03 e madrugada do dia 18/03/2013 houve chuvas torrenciais, que ocasionaram diversos impactos em áreas do litoral norte de São Paulo (SP), litoral sul, Baixada Fluminense e Região Serrana do Rio de Janeiro (RJ). A área mais castigada foi a serra fluminense, principalmente o município de Petrópolis, onde choveu em $24 \mathrm{~h}$ cerca de $400 \mathrm{~mm}$. Os deslizamentos de terra provocaram diversos danos materiais e vítimas fatais. Segundo a Defesa Civil do Estado, pelo menos 33 pessoas morreram e mais de 1000 ficaram desabrigadas.

Episódios de chuva forte na Região Sudeste geralmente estão associados com a atuação da Zona de Convergência do Atlântico Sul (Quadro, 1994; Sanches, 2002; Pinheiro et al, 2011; Andrade e Pinheiro, 2012), sistemas frontais (Seluchi, 2006; Seluchi e Chou, 2009; Dereczynski, 2009) ou com sistemas convectivos isolados (Teixeira e Satyamurty, 2007). Além disso, áreas serranas sofrem o efeito da topografia. Vários estudos numéricos e observacionais têm sido realizados como o propósito de compreender melhor a circulação atmosférica e a influência dos processos de superfície no regime de precipitação nas áreas de serra (Chou et al., 2006; Guimarães et al., 2006). Andrade e Pinheiro, 2011, usando dados do CPC (Climate Prediction Center/ NOAA) no período de 1960-1990, mostraram que valores acima de $40 \mathrm{~mm} /$ dia de precipitação representam 0,5\% da amostra em cidades da Região Serrana do Rio de Janeiro, ou seja, volumes superiores são considerados eventos extremos.

O objetivo deste trabalho é analisar a condição sinótica associada à chuva forte, bem como a destreza dos modelos numéricos, regional e global, em prever a precipitação. Será utilizado também um Método para Deteç̧ão de Padrões Atmosféricos - MedPat (Dolif e Nobre, 2012) para verificar se há semelhanças entre o evento analisado e algum padrão atmosférico de chuva forte ocorrido no passado.

\section{Dados e metodologia}

Para este trabalho foram usadas imagens do satélite GOES-12 no canal infravermelho, do dia 17 de março de 2013, dados observados de precipitação acumulada em 24 horas no dia 17 de março de 2013 do Instituto Nacional de Meteorologia (INMET) e do INEA (Instituto Estadual do Ambiente), perfil atmosférico obtido por radiossondagem do aeroporto do Galeão, na cidade do Rio de Janeiro e cartas sinóticas elaboradas pelo Grupo de Previsão de Tempo (GPT) do Centro de Previsão de Tempo e Estudos Climáticos (CPTEC). Com o intuito de avaliar a precipitação prevista foram utilizadas as saídas de previsões dos modelos regionais ETA (Mesinger 1984; Black, 1994), de diferenças finitase baseado na coordenada ETA (Mesinger et al., 1988) com resolução horizontal de $15 \mathrm{~km}$ e BRAMS (Freitas et al., 2009 e Moreira et al., 2013) com resolução horizontal de 20 $\mathrm{km}$, rodados operacionalmente no CPTEC, e do modelo global GFS, proveniente do NCEP (National Centers for Environmental Prediction).

A busca por padrão sinótico análogo em eventos passados é feita pelo Método de Detecção de Padrões Atmosféricos (MeDPAt) (Dolif e Nobre, 2012) .O MeDPAt possui um banco de eventos passados e os compara com os campos previstos pelos membros do modelo ETA40 ensemble. Quando essa comparação apresenta similaridade acima de um limiar pré-definido, então interpreta-se como havendo risco de uma nova ocorrência de extremo de chuva. O MeDPAt é também utilizado pelo Grupo de Previsão de Tempo (GPT) do CPTEC/INPE como ferramenta operacional diagnóstica e prognóstica.

\section{Resultados}

Na tabela 1 é apresentado o volume de chuva acumulado em um intervalo de 24 horas para cidades da Região Serrana do Rio de Janeiro. Na estação meteorológica operada pelo Instituto Estadual do Ambiente (INEA) e localizada no Bairro Quitandinha (Petrópolis) foi registrado mais de $400 \mathrm{~mm}$ de precipitação (Tabela 1). Acumulados superiores a $100 \mathrm{~mm}$ também foram registrados no litoral sul do RJ (região da Costa Verde), Baixada Fluminense e região de Macaé. No litoral norte de SP a chuva intensa também causou transtornos, levando o município de São Sebastião a decretar estado de calamidade pública.

Tabela 1: Acumulados de chuva em 24 horas observados em algumas estações meteorológicas.

\begin{tabular}{|c|c|c|}
\hline Cidade & $\begin{array}{c}\text { Chuva em 24h } \\
\text { Fonte: INEA }\end{array}$ & $\begin{array}{c}\text { Climatologia } \\
\text { Março } \\
\text { Fonte: Inmet }\end{array}$ \\
\hline Petrópolis & $424,5 \mathrm{~mm}$ & - \\
\hline Teresópolis & $213,6 \mathrm{~mm}$ & $263 \mathrm{~mm}$ \\
\hline Nova Frigurgo & $182,6 \mathrm{~mm}$ & $154,6 \mathrm{~mm}$ \\
\hline
\end{tabular}

\subsection{Análise Sinótica}

A sequência de imagens do satélite GOES-12 para o dia 17/03/2013 (Figura 1) mostra o deslocamento de áreas de instabilidade, que inicialmente se estendiam do Atlântico à costa norte de SP. 


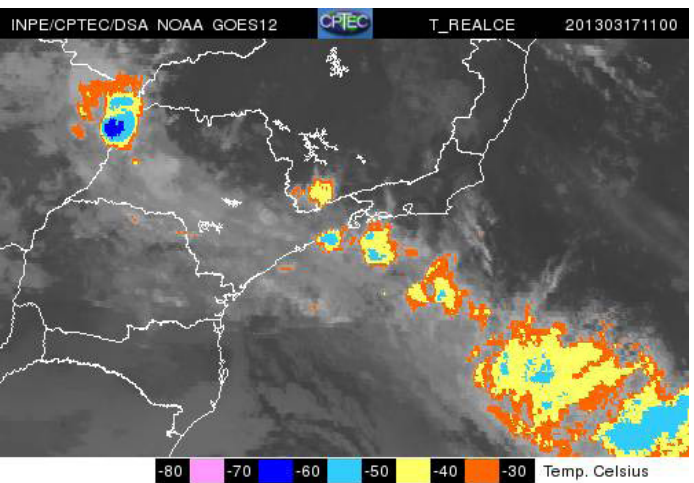

(a)

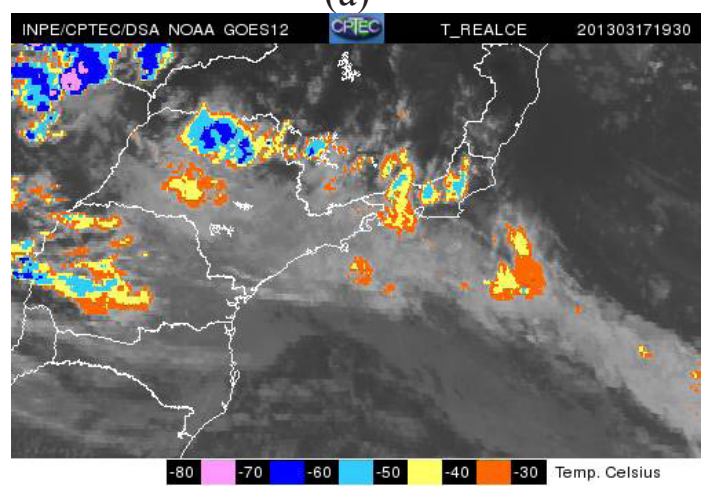

(c)

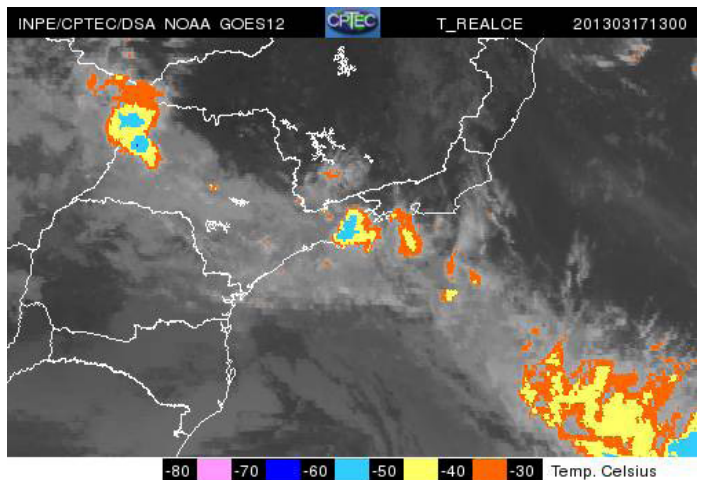

(b)

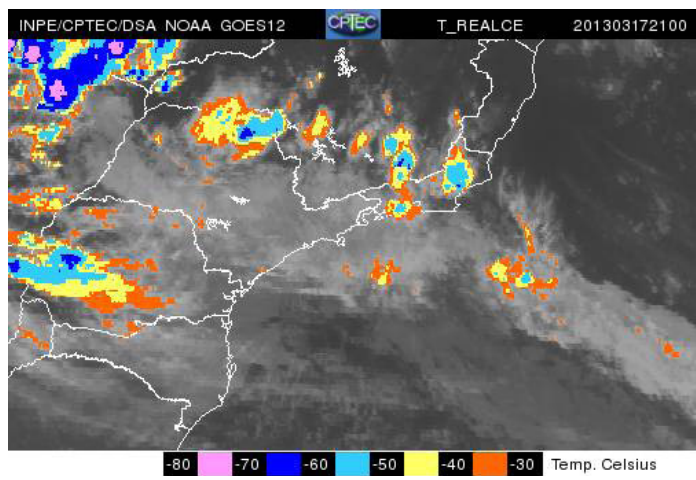

(d)

Figura 1: Sequência de imagens do satélite GOES12 no canal infravermelho e realçada para (a) 11:00 UTC, (b) 13:00 UTC, (c) 19:30 UTC e (d) 21:00 UTC do dia 17/03/2013.

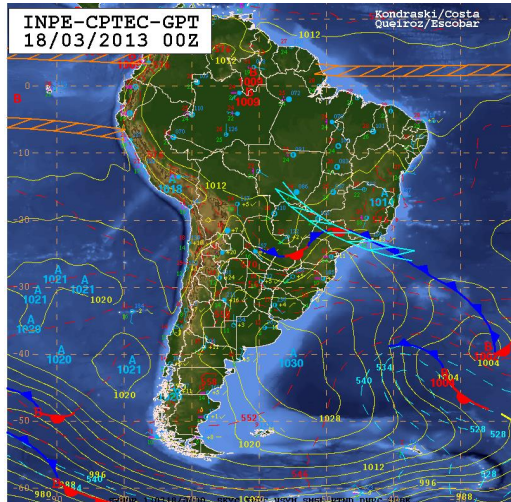

(a)

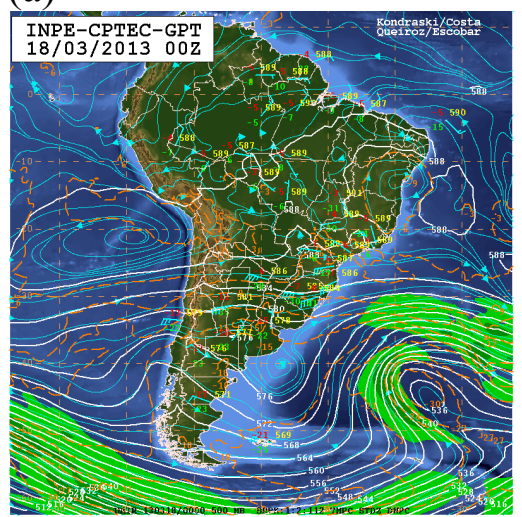

(c)

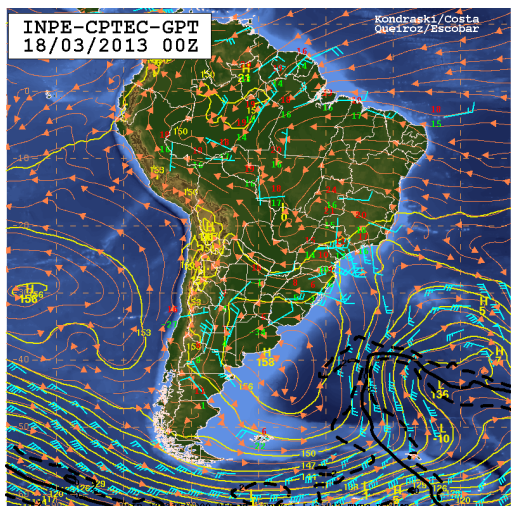

(b)

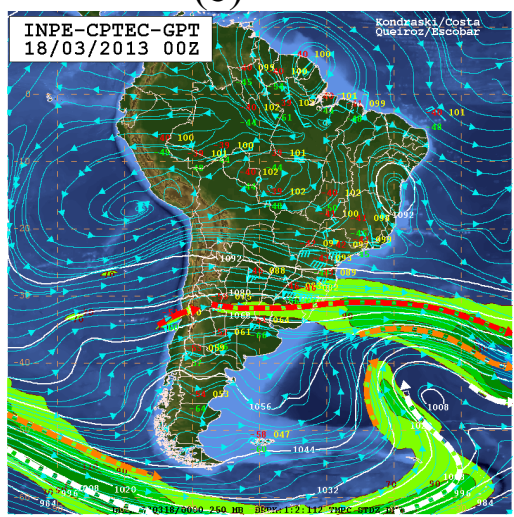

(d)

Figura 2: Cartas sinóticas de (a) superfície, (b) 850 hPa, (c) 500 hPae (d) 250 hPa para 00:00 UTC do dia 18/03/2013. 
Neste último setor, havia nebulosidade do tipo convectiva, que começou a se intensificar entre 09:00 e 11:00 UTC, alcançando temperaturas de topos entre $-50^{\circ} \mathrm{C}$ e $-60^{\circ} \mathrm{C}$. À medida em que a convecção inicial perdia força, novas células convectivas se organizavam na dianteira do sistema frontal, promovendo chuvas intensas sobre o litoral norte de SP. No decorrer da tarde deste dia (entre 1500 UTC e 1700 UTC), núcleos convectivos se formaram também sobre a costa sul do RJ, atingindo mais tarde a Região Serrana do Estado e a Baixada Fluminense. A persistência da convecção foi determinante para gerar grandes volumes de chuva em um intervalo relativamente curto de tempo. É interessante destacar que não houve registro de atividade elétrica significativa (dados não apresentados).

A análise da carta sinótica da 00:00 UTC do dia 18/03/2013 (21h local do dia 17) mostra uma frente fria na altura do RJ e um amplo anticiclone na sua retaguarda (Figura 2a). Este sistema frontal que chegou ao RJ foi bastante significativo para esta época do ano e provocou um intenso influxo de ar úmido sobre toda a costa de SP e do RJ. Os ventos perpendiculares à costa que aparecem na carta de $850 \mathrm{hPa}$ (Figura 2b) indicam um cenário favorável para chuvas intensas e contínuas. Nota-se que na troposfera média e alta (Figura 2c e 2d) não havia nenhum mecanismo dinâmico que pudesse intensificar a convecção. Essas características sugerem a presença do sistema frontal em baixos níveis como o principal deflagrador das chuvas torrenciais.

A Figura 3 mostra a sondagem do aeroporto de Galeão (Rio de Janeiro) da 00:00 UTC do dia 18/03. Observase um perfil praticamente saturado, com $63,76 \mathrm{~mm}$ de água precipitável. Através dos valores dos índices de instabilidade nota-se que não havia muita instabilidade atmosférica. Por outro lado, havia umidade em toda a coluna troposférica e o predomínio de ventos intensos do quadrante sul na camada mais baixa. A geografia da região coloca esses ventos aproximadamente perpendiculares à costa do RJ, contribuindo para aumentar a convergência de umidade devido à desaceleração do escoamento ao encontrar uma superfície com maior rugosidade do que o mar. Além disso, o escoamento de sul logo encontra uma barreira topográfica que é a Região Serrana do Rio de Janeiro, onde a convergência ganha maior intensidade e o ar ainda é forçado a subir devido à barreira natural formada pelas montanhas.

\subsection{Prognóstico do evento}

A habilidade dos modelos operacionais em simular a precipitação para este evento foi avaliada utilizando os modelos regionais ETA $(15 \mathrm{~km})$ e BRAMS $(20 \mathrm{~km})$ e o modelo global GFS (Global Forecast System). Para contemplar os eventos de chuva observados no litoral de SP e em parte do RJ foi avaliada a precipitação prevista em dois intervalos distintos.

Inicialmente, tem-se o acumulado previsto para o entre 00:00 UTC do dia 17/03 e 00:00 UTC do dia 18/03 (Figura 4). Nota-se que o posicionamento das chuvas foi bastante semelhante nos três modelos, com maior detalhamento nos modelos regionais. Observa-se que o BRAMS e o ETA indicavam os maiores volumes de chuva na costa sul de SP, com valores acima de $80 \mathrm{~mm}$ e $100 \mathrm{~mm}$, respectivamente. De acordo com a rede de estações meteorológicas disponíveis, o maior volume de chuva foi observado em São Sebastião, com $224 \mathrm{~mm}$ em 24 horas. Na costa sul de SP não houve registro de

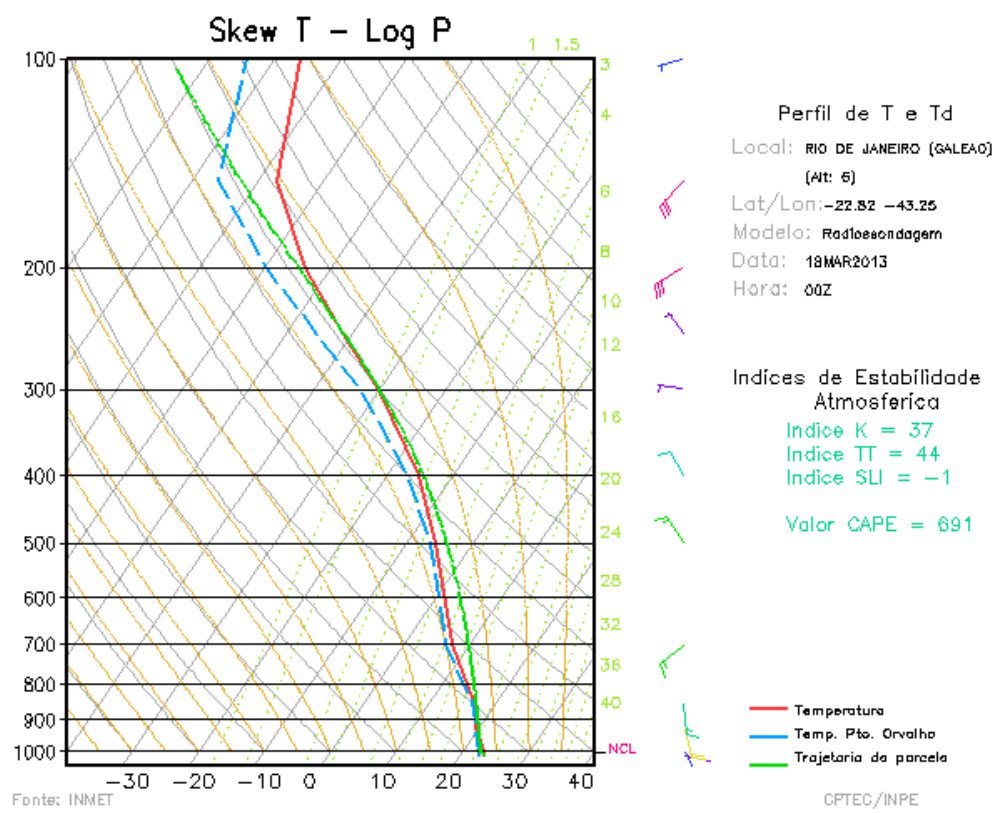

Figura 3: Sondagem do aeroporto de Galeão para 00:00 UTC do dia 18/03/2013. (Fonte: CPTEC-INPE; INMET) 
chuva significativa, o que mostra superestimativa das previsões naquela área.

Em relação à precipitação prevista para o entre 12:00 UTC do dia 17/03 e 12:00 UTC do dia 18/03 (Figura 5), percebe-se que os modelos já indicavam o posicionamento do máximo de chuva em direção ao RJ. Observa-se que o modelo ETA previa acumulados superiores a $80 \mathrm{~mm}$ entre o litoral norte de SP e litoral sul do RJ, enquanto o BRAMS indicava o máximo de chuva aproximadamente sobre a região metropolitana do RJ. O modelo GFS mostrava o máximo de chuva sobre o litoral norte de SP.
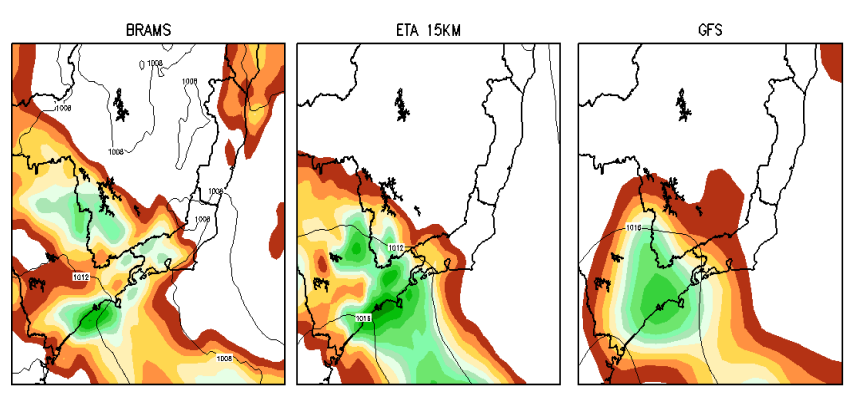

Figura 4: Precipitação em 24 horas prevista pelos modelos BRAMS, ETA15 e GFS, para o período entre 00:00 UTC do dia 17/03 e 00:00 UTC do dia 18/03/2013
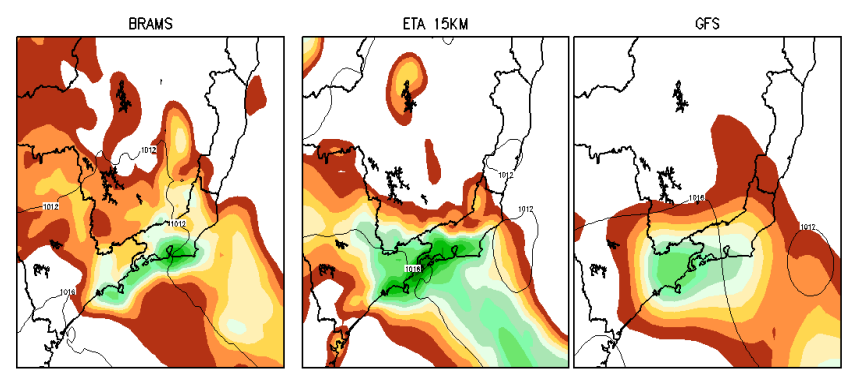

Figura 5: Idem Figura4 para o período entre 12:00 UTC do dia 17/03 e 12:00 UTC do dia 18/03/2013.

A Figura 6 mostra a distribuição espacial de umidade relativa em $900 \mathrm{hPa}$ e de vento meridional a 10 metros para o evento aqui analisado (esquerda) e para um outro evento extremos de chuva ocorrido em 02 de novembro de 2003. Isso mostra que o padrão sinótico do evento atual se assemelha ao padrão daquele de aproximadamente 10 anos atrás. O MeDPAt é executado em tempo real, à medida em que ficam prontas as previsões operacionais duas vezes por dia no CPTEC. Dessa forma é possível obter essas informações de similaridade antes da data com provável ocorrência do extremo de chuva e, assim, enviar um aviso meteorológico para que sejam mitigados os impactos negativos causados pelo excesso de chuva.

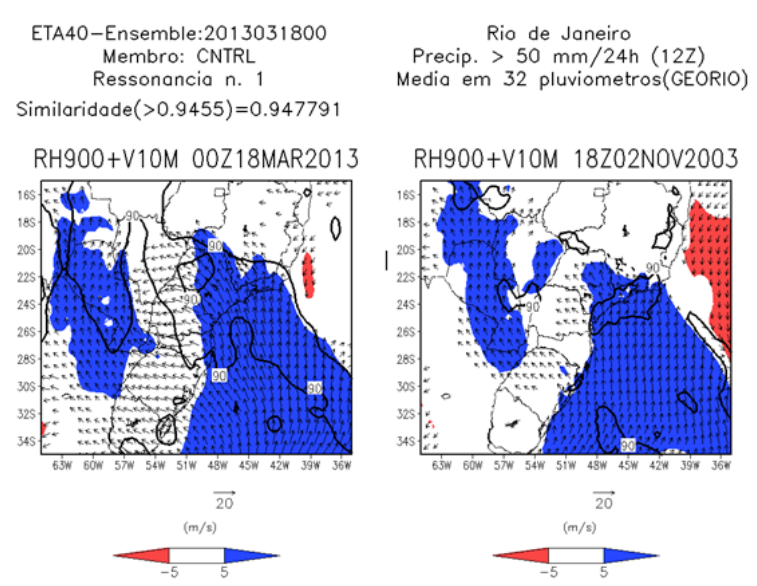

Figura 6: Distribuição espacial de umidade relativa em $900 \mathrm{hPa}$ (linha preta contínua) e de vento meridional a 10 metros. À esquerda tem-se o evento atual e à direita um evento ocorrido 10 anos antes.

\section{Conclusão}

Eventos extremos de chuva são um desafio para a meteorologia operacional. No caso analisado, a chuva foi causada pela passagem de uma frente fria acompanhada de intensos ventos em baixos níveis, favorecendo a convergência de umidade que resultou nas chuvas torrenciais. A orografia da Região Serrana do Rio de Janeiro e da Serra do Mar também parecem ter favorecido a intensificação da atividade convectiva.

O método objetivo (MeDPat) para detecção das características atmosféricas que favorecem a ocorrência de eventos extremos de chuva apontou semelhança do evento atual com outro ocorrido 10 anos antes. Isso mostra o potencial prognóstico do método, através da recorrência de características atmosféricas com a utilização das variáveis vento a 10 metros e umidade relativa em $900 \mathrm{hPa}$.

A coerência entre as previsões dos modelos numéricos, aliada ao reconhecimento pelo meteorologista de um padrão sinótico de alto risco, favoreceu a realização de um prognóstico eficiente. Por outro lado, a intensidade e a localização exata da precipitação ainda representam desafios para a meteorologia operacional, podendo ser superadas pela criação de estratégias e pela experiência do meteorologista previsor.

\section{Referências}

ANDRADE, K. M.; PINHEIRO, H. R. Análise sinótica e simulação numérica de um evento extremo de chuva sobre o litoral de São Paulo e do Rio de Janeiro em dezembro de 2011. In: XVII Congresso Brasileiro de Meteorologia, 2012, Gramado-RS. Anais...2012. DVD. Disponível em:<http:// www.cbmet2012.com/anais/pdfs/62FN.pdf $>$ Acesso em 
05 abr. 2013

ANDRADE, K. M.; PINHEIRO, H. R. Simulação de eventos extremos de precipitação na Região Serrana do Rio de Janeiro no clima presente e futuro utilizando o modelo ETA-HADCM3. In: Simpósio Internacional de Climatologia, 2011, João Pessoa- PB. Anais...2011. Disponível em: $<$ http://mtc-19. sid.inpe.br/col/sid.inpe.br/mtc-m19/2011/11.16.13.26/ doc/Andrade;\%20Pinheiro20Simula\%e7\%e3o\%20 de\%20Eventos.PDF> Acesso em 05 abr. 2013

BLACK, T. L. The new NMC mesoscale Eta Model: Description and forecast examples. Weather Forecasting, $v$. 9, p. 265-278, 1994.

CHOU, S. C.; BUSTAMANTE, J. F.; GOMES, J. L. Predictability of heavy rainfall events over the Serra do Mar. In: International Conference on Southern Hemisphere Meteorology and Oceanography, 08, Foz do Iguaçu (PR), Br. Proceedings... 2006. p. 1729-1731. CD-ROM. (INPE-13869PRE/9050).

DERECZYNSKI, C. P.; OLIVEIRA, J.S.;MACHADO, C. 0. Climatologia da precipitação no município do Rio de Janeiro. RBMET, v.24,n. 1, 24-38, 2009.

DOLIF, G.; NOBRE, C. A. Improving extreme precipitation forecasts in Rio de Janeiro, Brazil:are synoptic patterns efficient for distinguishing ordinary from heavy rainfall episodes? Amospheric Science Letters, v. *,2012. Doi: $<\underline{10.1002 / a s 1.385>}$

FREITAS, S. R.; LONGO, K. M.; SILVA DIAS, M. A. F.; CHATFIELD, R.; SILVA DIAS, P.; ARTAXO, P.; ANDREAE, M. O.; GRELL, G.; RODRIGUES, L. F.; FAZENDA, A.; PANETTA, J. The Coupled Aerosol and Tracer Transport model to the Brazilian developments on the Regional Atmospheric Modeling System (CATT-BRAMS) - Part 1: Model description and evaluation. Atmospheric Chemistry and Physics, v. 9, n. 8, p. 2843-2861, 2009.

GUIMARÃES, J. M.; DERECZYNSKI, C. P.; CHOU, S. C. Avaliação do Modelo Eta durante um Episódio de Chuvas Intensas na Região da Serra do Mar. Congresso Brasileiro de Meteorologia, XIV - (CBMET), Florianópolis, SC. Anais...2006. CD-ROM.

MESINGER F. A blocking for representation of mountains in atmospheric models. Rivista di Meteorologia Aeronautica, v.44, n. 1-4, p.195-202, 1984.

MESINGER F.; JANJIC, Z.I.; NICKOVIC, S.; GAVRILOV, D.; DEAVEN, D.G. The step-mountain coordinate: model description and performance for cases of Alpine lee cyclogenesis and for a case of Appalachian redevelopment. Mon. Wea. Rev., v. 116, p. 1493-1518, 1988.
MOREIRA, D. S.; FREITAS, S. R.; BONATTI, J. P.; MERCADO, L. M.; ROSÁRIO, N. M. É.; LONGO, K. M.; MILLER, J. B.; GLOOR, M.; GATTI, L. V. Coupling between the JULES land-surface scheme and the CCATT-BRAMS atmospheric chemistry model (JULES-CCATT-BRAMS1.0): applications to numerical weather forecasting and the CO2 budget in South America. Geoscientific Model Development Discussions, v. 6, n. 1, p. 453-494, 2013.

PINHEIRO, H. R.; ANDRADE, K. M, MOURA, C.W. A maior catástrofe climática do Brasil sob a visão operacional do CPTEC/INPE. In: Simpósio Internacional de Climatologia, 2011, João Pessoa-PB. Anais...2011. DVD.

QUADRO, M. F. L. Estudo de episodios de zonas de convergencia do Atlantico Sul (ZCAS) sobre a America do Sul. 1994. 123 p. (INPE-6341-TDI/593). Dissertação (Mestrado em Meteorologia) - Instituto Nacional de Pesquisas Espaciais,

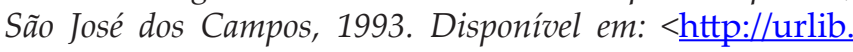
net/sid.inpe.br/MTC-m13@80/2005/08.26.14.10> Acesso em 26 mar. 2013

SANCHES, M. B. Análise Sinótica da Zona de Convergência do Atlântico Sul (ZCAS) Utilizando-se a Técnica de Composição. São José dos Campos. Dissertação (Mestrado em Meteorologia) - Instituto Nacional de Pesquisas Espaciais, 2002.

SELUCHI, M. E. Padrões sinóticos associados a situações de deslizamentos de encostas na serra do mar. Congresso Brasileiro de Meteorologia, XIV - (CBMET), Florianópolis, SC. Anais...2006. CD-ROM.

SELUCHI, M. E., E S. C. CHOU. Synoptic patterns associated with landslide events in the Serra do Mar, Brazil. Theoretical and Applied Climatology, 98, 67-77, 2009.

TEIXEIRA, M. S.; SATYAMURTY, P. Dynamical and synoptic characteristics of heavy rainfall episodes in southern Brazil. Mon. Wea Rev., v. 135, p. 598-617, 2007. 